\title{
Performance Evaluation of Binary Negative-Exponential Backoff Algorithm in Presence of a Channel Bit Error Rate*
}

\author{
Bum-Gon Choi, Hyung Joo Ki, Min Young Chung ${ }^{\star \star}$, and Tae-Jin Lee \\ School of Information and Communication Engineering \\ Sungkyunkwan University \\ 300, Chunchun-dong, Jangan-gu, Suwon, Kyunggi-do, 440-746, Korea \\ \{gonace, ki0724, mychung, tjlee\}@ece.skku.ac.kr
}

\begin{abstract}
The IEEE 802.11 standard basically uses a DCF (Distributed Coordination Function) to access a wireless channel. However, the DCF uses wireless resource ineffectively when there are many contending stations and a high bit error rate. To enhance the performance of the wireless LAN, Ki et al. proposed a Binary Negative-Exponential Backoff (BNEB) algorithm. We found that the performance of the BNEB algorithm was better than the conventional DCF. However, erroneous channel environment was not considered. In our work, we propose an analytical model for the BNEB algorithm in the presence of transmission error and compare the performance of the DCF with the BEB algorithm to that with the BNEB algorithm. From the result, the BNEB algorithm yields better performance than the $\mathrm{DCF}$ when the bit error rate $(B E R) \leq 10^{-5}$.
\end{abstract}

Keywords: DCF, BNEB, BER, Contention Window.

\section{Introduction}

The IEEE 802.11 medium access control (MAC) employs the distributed coordination function (DCF) [1]. The DCF is a contention-based channel access function adopting a carrier sense multiple access with collision avoidance (CSMA/CA) for frame transmission during the contention period. However, in the DCF, the more the number of stations uses the wireless resources, the more collision occurrences are possible. To solve these problems, much research on the performance of the IEEE 802.11 DCF has been conducted.

Bianchi presented an analytical model and showed that the proposed model was very accurate [2] [3. The performance of the DCF in the presence of transmission error was evaluated in [4] [5]. For effective management of wireless resources,

\footnotetext{
* This work was supported by a grant No.R01-2006-000-10402-0 from the Basic Research Program Korea Science and Engineering Foundation of Ministry of Science \& Technology and by the MIC(Ministry of Information and Communication), Korea, under the ITRC(Information Technology Research Center) support program supervised by the IITA(Institute of Information Technology Assessment), IITA-2006(C1090-0603-0046).

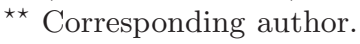


Ki et al. proposed a Binary Negative-Exponential Backoff (BNEB) algorithm [6]. The BNEB algorithm increases the contention window to the maximum window size when stations experience a collision and decreases the contention window size by half when a transmission is successful. In [6], the results showed that the BNEB had better performance than the DCF with the BEB algorithm in ideal channel conditions. However, the performance of the BNEB algorithm may depend on the presence of transmission error. Thus, we propose an analytical model for the BNEB algorithm and evaluate the performance of the BNEB algorithm in presence of transmission error.

The rest of this paper is organized as follows. Section 2 describes an analytical model of the BNEB algorithm in the presence of transmission error under the saturation condition. In Section 3, we describe our verification of our analytical model by simulations and compare the throughput of the DCF with the BNEB algorithm to that with BEB algorithm. Finally, we conclude in Section 4

\section{Analytical Model for BNEB}

Our analysis assumes that there are $\mathrm{n}$ stations having frame(s) to transmit and each station has frame(s) after successful transmission. In addition, it is possible that a station fails to transmit a frame because of channel noise. For a station, $s(t)$ and $b(t)$ are defined as the random process representing the backoff stage and the backoff counter at time $t$. The BNEB algorithm can be modeled as a bi-dimensional discrete-time Markov chain $(s(t), b(t))$. Let $b_{i, j}=$ $\lim _{t \rightarrow \infty} P\{s(t)=i, b(t)=j\}$ and $p$ be the transmission failure probability that a station experiences a collision or transmission error in a slot. Then, the state transition probabilities and state relations are given in [6]. And $p$ is given by:

$$
p=1-(1-\tau)^{n-1}(1-B E R)^{l+H},
$$

where the $B E R, l$ and $H$ represent the channel bit error rate, packet payload size and the packet header size.

From [6] and $p$, we can obtain $b_{0,0}$ :

$$
b_{0,0}=\frac{1}{\frac{(W p+1)+\frac{1-p}{2}\left[W\left(\frac{1-p}{2}\right)^{m}-1\right]}{p(1+p)}+\frac{W+1}{2}\left(\frac{1-p^{L}}{1-p}\right)} .
$$

Let $\tau$ be the probability of attempting to transmit a frame. Then we have

$$
\tau=\sum_{i=-m}^{L} b_{i, 0}=\left(\frac{1}{p}+\frac{1-p^{L}}{1-p}\right) b_{0,0} .
$$

Using $\tau$, a transmission success probability $P_{s}$, a collision probability $P_{c}$ and a transmission error probability $P_{e r}$ are calculated as

$$
P_{s}=\frac{n \tau(1-\tau)^{n-1}}{P_{t r}}(1-P E R)
$$




$$
\begin{gathered}
P_{c}=1-\frac{n \tau(1-\tau)^{n-1}}{P_{t r}}, \\
P_{e r}=\frac{n \tau(1-\tau)^{n-1}}{P_{t r}} P E R,
\end{gathered}
$$

where $P E R$ is the packet error rate and $P_{t r}$ is the probability that there is at least one transmission.

$$
P_{t r}=1-(1-\tau)^{n}
$$

Finally, we can obtain the normalized saturation throughput of the BNEB algorithm in the presence of a channel bit error rate as follows.

$$
S=\frac{P_{t r} P_{s} l}{\left(1-P_{t r}\right) \sigma+P_{t r} P_{s} T_{s}+P_{t r} P_{c} T_{c}+P_{t r} P_{e r} T_{e r}},
$$

where $\sigma$ is the duration of a backoff slot, $T_{s}, T_{c}$ and $T_{e r}$ are the average time intervals that the medium is sensed busy due to a successful transmission, a collision or an error transmission, respectively.

\section{Performance Evaluations}

To evaluate the performance of the DCF with the BEB and BNEB algorithms in the presence of a transmission error, we used the table in [6] for the MAC parameters. The normalized saturation throughput of the DCF with the BEB and BNEB algorithms is shown in Figure 1. Figure 1(a) illustrates that the analytical results of the BNEB algorithm are close to the simulation results and depicts the throughput difference between the DCF with the BEB and BNEB algorithms as the $B E R$ increases. In the result, the number of contending stations does not affect the saturation throughput of the BNEB algorithm in contrast with the DCF with the BEB algorithm when $B E R \leq 10^{-5}$. When $B E R>10^{-5}$, the possibility of transmission failure by a transmission error is larger than that of transmission failure by a collision. Therefore, the difference of the DCF with the BEB and BNEB algorithms is decreased and the throughput of both algorithms reaches to 0 as $B E R$ increases.

Figure 1(b) shows the saturation throughput of the DCF with the BEB and BNEB algorithms as the number of contending stations increases when the $B E R$ is fixed at $10^{-4}, 10^{-5}$ and $10^{-6}$. When there are many contending stations, because the collision is more frequent than the transmission error, the BNEB algorithm shows better performance than the DCF. However, the backoff time strongly affects the saturation throughput of two algorithms when there are less contending stations. In the small number of contending stations and high $B E R$, since the backoff time of the BNEB algorithm is larger than that of the DCF with the BEB algorithm, the performance of the BNEB algorithm is less than that of the DCF with the BEB algorithm. However, because most wireless devices operate where $B E R \leq 10^{-5}[7$, in most cases, the performance of the BNEB algorithm is better than that of the DCF with the BEB algorithm. 


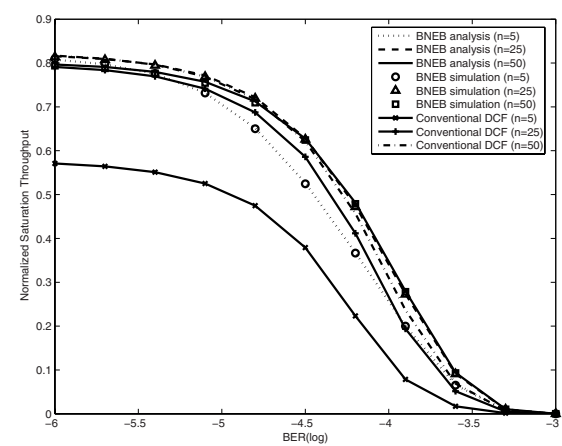

(a) varying channel $B E R$.

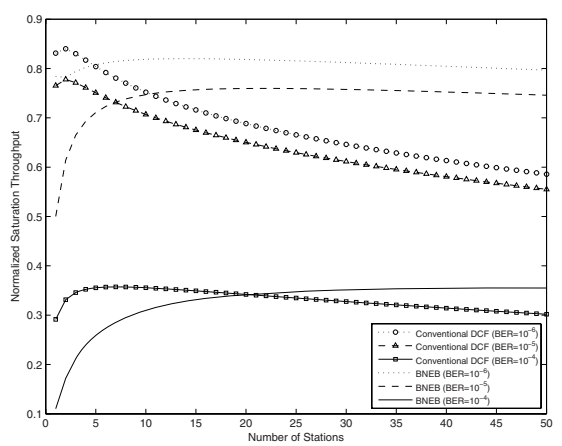

(b) variable number of stations.

Fig. 1. Comparisons of normalized saturation throughput between the DCF with the $\mathrm{BEB}$ and BNEB algorithms

\section{Conclusion}

In this paper, we described our evaluation of the performance of a Binary Negative-Exponential Backoff (BNEB) algorithm in the presence of transmission error by means of an analytical model and simulations of the saturation condition. With low BER, the performance of the BNEB algorithm was better than the DCF with the BEB algorithm because of effective collision resolution. Because of ineffective management of backoff time, the throughput of the BNEB algorithm was smaller than DCF with BEB algorithm with high $B E R$. However, we can expect that the BNEB algorithm will show better performance than DCF because most wireless devices operate when $B E R \leq 10^{-5}$.

\section{References}

1. IEEE standard for Wireless LAN Medium Access Control (MAC) and Physical Layer (PHY) Specifications. ISO/IEC 8802-11: (1999(E)) Aug. 1999

2. Bianchi, G.: IEEE 802.11-Saturation Throughput Analysis. IEEE Communications Letters, Vol. 2, No. 12, (1998) 318-320.

3. Bianchi, G.: Performance Analysis of the IEEE 802.11 Distributed Coordination Function. IEEE Journal on Selected Areas in Communications, Vol. 18, No. 3, (2000) 535-547.

4. Chatzimisios, P., Boucouvalas, A. C., Vitsas, V.: Performance Analysis of IEEE 802.11 DCF in Presence of Transmission Errors. Proceedings of IEEE International Conference on Communications, Vol. 7, (2004) 3854 - 3858.

5. Chatzimisios, P., Boucouvalas, A. C., Vitsas, V.: Influence of Channel BER on IEEE 802.11 DCF. Electronics Letters, Vol. 39, No. 23, (2003) 1687 - 1689.

6. Ki, H. J., Choi, S.-H., Chung, M. Y., Lee, T.-J.: Performance Evaluation of Binary Negative-Exponential Backoff Algorithm in IEEE 802.11 WLAN. MSN 2006, LNCS 4325, 294-303.

7. http://support.dell.com/support/edocs/network/p62005/en/specs.htm 